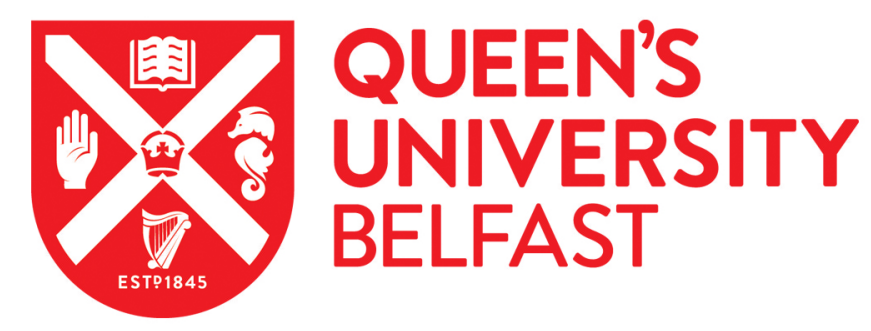

\title{
EGFR-targeted immunoliposomes efficiently deliver docetaxel to prostate cancer cells
}

Eloy, J. O., Ruiz, A., de Lima, F. T., Petrilli, R., Raspantini, G., Nogueira, K. A. B., Santos, E., de Oliveira, C. S., Borges, J. C., Marchetti, J. M., Al-Jamal, W. T., \& Chorilli, M. (2020). EGFR-targeted immunoliposomes efficiently deliver docetaxel to prostate cancer cells. Colloids and Surfaces B: Biointerfaces, 194, [111185]. https://doi.org/10.1016/j.colsurfb.2020.111185

Published in:

Colloids and Surfaces B: Biointerfaces

Document Version:

Peer reviewed version

Queen's University Belfast - Research Portal:

Link to publication record in Queen's University Belfast Research Portal

Publisher rights

Copyright 2020 Elsevier

This manuscript is distributed under a Creative Commons Attribution-NonCommercial-NoDerivs License

(https://creativecommons.org/licenses/by-nc-nd/4.0/), which permits distribution and reproduction for non-commercial purposes, provided the author and source are cited.

\section{General rights}

Copyright for the publications made accessible via the Queen's University Belfast Research Portal is retained by the author(s) and / or other copyright owners and it is a condition of accessing these publications that users recognise and abide by the legal requirements associated with these rights.

Take down policy

The Research Portal is Queen's institutional repository that provides access to Queen's research output. Every effort has been made to ensure that content in the Research Portal does not infringe any person's rights, or applicable UK laws. If you discover content in the Research Portal that you believe breaches copyright or violates any law, please contact openaccess@qub.ac.uk. 


\section{EGFR-targeted immunoliposomes efficiently deliver docetaxel to prostate cancer cells}

Josimar O. Eloy ${ }^{1 *}$, Amalia Ruiz ${ }^{2}$, Felipe Tita de Lima ${ }^{3}$, Raquel Petrilli ${ }^{4}$, Giovanni Raspantini ${ }^{5}$, Karina Alexandre Barros Nogueira ${ }^{1}$, Elias Santos ${ }^{1}$, Carlos Sabino de Oliveira ${ }^{6}$, Júlio César Borges $^{6}$, Juliana Maldonado Marchetti ${ }^{5}$, Wafa T. Al-Jamal ${ }^{2}$, Marlus Chorilli ${ }^{3}$

${ }^{1}$ Federal University of Ceará, College of Pharmacy, Dentistry and Nursing, Department of Pharmacy, Fortaleza, Ceará, Brazil

${ }^{2}$ School of Pharmacy, Queen’s University Belfast, 97 Lisburn Rd, Belfast BT9 7BL, UK

${ }^{3}$ School of Pharmaceutical Sciences of Araraquara - São Paulo State University, UNESP, Department of Drugs and Medicines, Araraquara, SP, Brazil

${ }^{4}$ University for International Integration of the Afro-Brazilian Lusophony, Institute of Health Sciences, Ceará, Brazil

${ }^{5}$ College of Pharmaceutical Sciences of Ribeirão Preto, University of São Paulo, Ribeirão Preto, São Paulo Brazil.

${ }^{6}$ São Carlos Institute of Chemistry, University of São Paulo, São Carlos, São Paulo, Brazil.

Short statistical summary of the article

Total words of manuscript body: 5860

Total Tables: 1

Total Figures: 7

*Corresponding author: Josimar O. Eloy, PhD. Department of pharmacy - Federal University of Ceará. Rua Pastor Samuel Munguba, 1210, Fortaleza - CE, Brazil. josimar.eloy@ufc.br 


\begin{abstract}
Prostate cancer is the second cause of cancer death in men worldwide. Docetaxel (DTX), an antimitotic drug, is widely used for the treatment of metastatic prostate cancer patients. Taxotere $^{\circledR}$ is a commercial DTX formulation. It contains a polysorbate 80 surfactant to improve DTX aqueous solubility, which has been associated with hypersensitivity reactions in patients. Liposomes have been used as promising delivery systems for a range of hydrophobic drugs, such as DTX, offering improved drug water solubility and biocompatibility, without compromising its anticancer activity. Herein, DTX-loaded liposomes were developed using the Box-Behnken factorial design. The optimized formulation was nano-sized, homogenous in size $(67.47 \mathrm{~nm})$ with high DTX encapsulation efficiency (99.95\%). The encapsulated DTX was in a soluble amorphous state, which was slowly released. Next, to increase the liposomes selectivity to prostate cancer cells, cetuximab, an anti-EGFR monoclonal antibody. was successfully conjugated to the surface of liposomes, without compromising cetuximab protein structure and stability. As expected, our results showed higher cellular uptake and toxicity of immunoliposomes, compared to non-targeted liposomes, in DU145 (EGFRoverxpressing) prostate cancer cells. To the best of our knowledge, this is the first report of engineering EGFR-targeted liposomes to enhance the selectivity of DTX delivery to EGFR-positive prostate cancer cells.
\end{abstract}

Keywords: docetaxel, EGFR, cetuximab, immunoliposomes, prostate cancer. 


\section{INTRODUCTION}

Prostate cancer is the second most common cancer in men worldwide, with a record of 1.276.106 new cases in 2018, as well as 358.989 death (Rawla 2019). There are cases in which the neoplasm progresses and selective mutations occur that result in metastatic castration-resistant prostate cancer (mCRPC), in other words, the disease becomes refractory to androgen deprivation therapy (Tucci et al. 2019; Nelson et al., 2020.).

Docetaxel (DTX) is a first-line drug for the treatment of mCRPC. It is a semi-synthetic derivative of 10-deacetylbaccatin III, a precursor extracted from the branches of the European yew (Taxus baccata). This drug belongs to the class of taxanes and its mechanism of action is based on the binding to beta-tubulin to promote the stabilization of microtubules during the process of mitosis, thus, the dynamics of microtubules is inhibited and cell division is interrupted. The formulation containing DTX (Taxotere ${ }^{\circledR}$, Sanofi-Aventis, Paris, France) has demonstrated clinical efficacy (Engels, Mathot, and Verweij 2007; Saad and Miller 2014). However, there are several adverse effects related to drug toxicity. The most significant effect is the occurrence of neutropenia, anemia, gastrointestinal disorders and peripheral neuropathy (Mager et al. 2019). In addition, the presence of polysorbate 80 as a surfactant to solubilize the drug, which is highly lipophilic, can induce hypersensitivity reactions in patients (Engels, Mathot, and Verweij 2007).

To overcome these hurdles, DTX can be loaded into nanocarriers for better therapeutic outcomes. Among the various nanocarriers, liposomes have been widely studied. Liposomes are vesicles composed of one or more concentric lipid bilayers, which can encapsulate lipophilic and hydrophilic substances. They are biodegradable with low immunogenicity. Liposomes allow the protection of the drug, reducing physiological degradation and thus increasing bioavailability, controlling release and directing the therapy to have greater tissue selectivity ( $\mathrm{Li}$ et al. 2019).

Taking advantage of the passive accumulation of nanocarriers at the tumor environment, it is possible to increase the levels of cell internalization and ensure that the drug interacts with its site of action, through active targeting of these liposomes, by inserting ligands on their surface. When an antibody is conjugated to the liposomes, immunoliposomes originate, with high affinity to surface receptors on tumor cells. The interaction between the ligand and the receptor leads to ligand-receptor complex formation, which triggers endocytosis, followed by intracellular drug release (Merino, Zalba, and Garrido 2018).

Epidermal growth factor receptor (EGFR) is highly expressed in some prostate adenocarcinomas. EGFR is a transmembrane receptor with tyrosine kinase activity that stimulates tumor cell proliferation, angiogenesis, invasion and metastasis through the activation of several signaling pathways. Thus, it is possible to target DTX-loaded liposomes to EGFR, using an antiEGFR chimeric-murine monoclonal antibody (cetuximab) conjugated to the liposome containing docetaxel, so that this antibody competitively binds to the EGFR extracellular domain preventing 
the activation of the receptor by endogenous ligands and induces the internalization of the immunoliposome. Previously, docetaxel has been combined as free drug with cetuximab and an anti-angiogenic drug, sunitinib. Studies conducted in PC3 xenografts revealed that there were significantly higher pro-apoptotic effects observed for the sunitinib-docetaxel and sunitinibdocetaxel-cetuximab. Although DTX-loaded liposomes have been previously functionalized with trastuzumab for breast cancer therapy, to our knowledge this is the first report of anti-EGFR, DTX-loaded liposomes that would hypothetically assure the selective delivery of DTX to the prostate cancer cell, preventing a series of adverse effects and increasing the effectiveness of the treatment (Malmberg, Tolmachev, and Orlova 2011; Joshi et al. 2015; Guardiola, Varese, and Sa 2019; Rodallec et al. 2018; Guerin et al., 2008). We previously developed immunoliposomes, but for paclitaxel and rapamycin co-delivery, for another target, HER2 with a different monoclonal antibody, trastuzumab, for breast cancer (Eloy et al., 2017). For docetaxel delivery, immunoliposomes have been developed, with trastuzumab, for breast cancer (Rodallec et al., 2018). Regarding anti-EGFR immunoliposomes, we have previously loaded the hydrophilic 5fluorouracil for skin cell carcinoma using a different lipid composition, based on DSPC, cholesterol and DSPE-PEG-maleimide (Petrilli et al., 2018). For docetaxel delivery to EGFR, the present study is the first report on immunoliposomes, which is very important, considering that EGFR is highly expressed in some prostate adenocarcinomas, and the clinical relevance of combining both docetaxel and cetuximab in metastatic castration-resistant prostate cancer has been previously studied for free drugs in a phase II clinical trial (Cathomas et al., 2012). In terms of formulation development, for DTX and EGFR, only polymeric nanoparticles have been developed, and not for prostate, but for lung cancer (Patel et al., 2018). Therefore, we believe that the present work is innovative and represents an important contribution toward the development of a selective DTX formulation for aggressive types of prostate cancer.

The present work describes the engineering of anti-EGFR immunoliposomes containing DTX to treat prostate cancer. To achieve this, the Box-Benken model was used as a tool to optimize the liposomal formulation, regarding size, polydispersity and DTX encapsulation efficiency. Solid state physicochemical characterization was employed to determine the state of encapsulated drug. Next, anti-EGFR antibody was successfully chemically conjugated to the liposomal surface using maleimide chemistry. The integrity of the antibody following conjugation was determined using electrophoresis and fluorescence microscopy. Subsequently, the in vitro release profile and the colloidal stability were determined. Finally, the cytotoxicity and cellular uptake by confocal microscopy and flow cytometry were studied in low and high EGFRexpressing prostate cancer cells. 


\section{MATERIAL AND METHODS}

\subsection{Material}

Lipids soy phosphatidylcholine (SPC) and 1,2-distearoyl-sn-glycero-3phosphoethanolamine-N-[amino(polyethylene glycol)-2000] (DSPE-PEG2000) were purchased from Lipoid (Lipoid GmbH, Germany). Cholesterol (CHOL) and 1,2-distearoyl-sn-glycero-3phosphoethanolamine-N [maleimide(polyethylene glycol)-2000] (ammonium salt) (DSPE-PEGMal) were purchased from Sigma Aldrich Co. (St. Louis, MO, USA) and LaySan Bio (Arab, AL, USA), respectively. Docetaxel (DTX) was supplied by APIChem (China). Erbitux $5 \mathrm{mg} / \mathrm{mL}$ (Cetuximab) was obtained from Merck Serono (Biberach, Germany). Disposable PD-10 desalting columns and CL-4B were obtained from GE Healthcare (Pittsburgh, PA, USA). 3,3'dioctadecyloxacarbocyanine perchlorate (DiO), 1,1'-dioctadecyl-3,3,3',3'tetramethylindocarbocyanine perchlorate (DiI), paraformaldehyde, dialysis membrane molecular weight cut-off 14,000 Da and Traut's reagent were purchased from Sigma Aldrich Co. (St. Louis, MO, USA). ProLong Diamond with DAPI was purchased from Life Technologies (Carlsbad, CA, USA). Propidium iodide was purchased from Thermo Scientific (Pittsburgh, PA, USA). Precision Plus Dual Color Protein was obtained from Biorad.

\subsection{Liposomes development and characterization}

\subsubsection{Liposomes development and factorial design}

Liposomes were prepared with soy phosphatidylcholine (SPC), cholesterol (CHOL) and 1,2-distearoyl-sn-glycero-3-phosphoethanolamine-N-[amino(polyethylene glycol)-2000] (DSPE-PEG2000), with the inclusion of DTX in different concentrations . The preparation of the liposomes followed the classic hydration technique of the lipid film previously described (Eloy et al. 2016). The lipids and the drug were dissolved in $2 \mathrm{~mL}$ chloroform, which was removed under reduced pressure in a rotary evaporator so that a thin film remains after $30 \mathrm{~min}$ at $65{ }^{\circ} \mathrm{C}$, and then hydrated for $30 \mathrm{~min}$ at $150 \mathrm{rpm}$ using phosphate buffer ( $\mathrm{pH} 7.4$ ) as aqueous phase. Then, the formulations were subjected to homogenization in a high-pressure homogenizer (Avestin), at $1000 \mathrm{bar}$, for $30 \mathrm{~min}$. The non-encapsulated drug in suspension was separated by $0.45 \mu \mathrm{m}$ filtration and the non-encapsulated drug soluble in the buffer was separated by gel chromatography on a CL-4B column filtration.

In the present study, a Box-Behnken statistical design with three-factor, three-level was used to evaluate influence of independent variables (Lipid: drug molar ratio, CHOL lipid molar ratio and DSPE-PEG:lipid molar ratio) on the physicochemical characteristics of the liposomes. The experimental design consists of a group of points located at the midpoint of each edge and one point in the center of a cube. This approach allows the construction of second order 
polynomial models for the dependent variables that can provide theoretical value of an output. The polynomial equation generated by this statistical design (using MiniTab ${ }^{\circledR} 16.0$ software) is as follows (Equation 1) (Solanki, Parikh, and Parikh 2007):

$$
\begin{gathered}
Y_{i}=b_{0}+b_{1} X_{1}+b_{2} X_{2}+b_{3} X_{3}+b_{12} X_{1} X_{2}+b_{13} X_{1} X_{3}+b_{23} X_{2} X_{3}+b_{11} X_{1}^{2}+b_{22} X_{2}^{2}+ \\
b_{33} X_{3}^{2}
\end{gathered}
$$

(Equation 1)

Where $Y_{i}$ is the dependent variable; $b_{0}$ is the intercept; $X_{1}, X_{2}$ and $X_{3}$ are the independent variables and $b_{1}$ to $b_{33}$ are the regression coefficients.

Basically, 15 formulations were selected to evaluate the effects between the independent variables applied with desirability approach in order to optimize the preparation of liposomes where the responses such as particle size and polydispersity index value were targeted to the minimum, and encapsulation efficiency was targeted to the maximum. The desirability function (D) can vary over the range 0 to 1 for each response. The result of $\mathrm{D}$ equal to 0 indicates the undesirable value of the response and if the maximized value of $\mathrm{D}$ is found, the optimal conditions for the variables are obtained (Das and Mishra 2017).

\subsubsection{Nanoparticles characterization}

\subsubsection{Particle size, polydispersity and zeta potential}

Nanoparticles size and the polydispersity index (PdI) were determined at $25^{\circ} \mathrm{C}$ by dynamic light scattering measures, using HeNe laser operating at $4 \mathrm{~mW}$ and $633 \mathrm{~nm}$ wavelength. The equipment performs non-invasive measurements by "backscatteroptics" (NIBS), made at a detection angle of $173^{\circ}$ and the measurement position of the cuvette is automatically determined by the equipment's software. The equipment makes an average of 12 determinations for each analysis. The dispersions were diluted in Milli Q water (10-fold) and placed in cells of $1 \mathrm{~cm}$ of optical path. Zeta potential was determined by the electrophoretic mobility of the scattered particles subjected to an electric field.

\subsubsection{Encapsulation efficiency}

For the analysis of the encapsulation efficiency, liposome (without non-encapsulated drug or total fraction) was diluted in acetonitrile, filtered using a PTFE $0.45 \mu \mathrm{m}$ and analyzed by the analytical method. Briefly, for the quantification of DTX in nanoparticles, a validated high performance liquid chromatography (HPLC) method was used, with a mobile phase composed of acetonitrile: water: methanol, 65:25:15 (v/v), under a flow of $0.8 \mathrm{~mL} \mathrm{~min}^{-1}$, in a C18 reverse 
phase column ( $4.6 \times 250 \mathrm{~mm}, 5 \mu \mathrm{m})$ with injection of $20 \mu \mathrm{L}$ at $25^{\circ} \mathrm{C}$ and a wavelength of 232 $\mathrm{nm}$. The loading percentage was calculated according to Equation 2.

$$
\text { Loading percentage }=\frac{\text { amount of drug in the lipsomal fraction }(\mu g)}{\text { amount of drug in the total fraction }(\mu g)} \times 100 \quad \text { (Equation 2) }
$$

\subsubsection{Colloidal stability and lyophilization study}

In order to assess the nanoparticles stability, liposomes were analyzed for their colloidal stability. The samples, freeze-dried or not, were kept in a $2-8^{\circ} \mathrm{C}$ refrigerator and analyzed at intervals of up to 60 days. For lyophilized samples, sucrose was used as a cryoprotectant in different lipid: sucrose molar ratios (1:10; 1:20 and 1:30). These samples were frozen overnight in an ultra-freezer at $-80^{\circ} \mathrm{C}$ and then lyophilized for 48 hours. The stability was monitored by particle size, PdI and zeta potential.

\subsubsection{Fourier transform infrared spectroscopy (FTIR)}

Samples of liposomes previously lyophilized with sucrose (lipid to sugar molar ratio, 1:20) were mixed with potassium bromide and compressed in a hydraulic press. The scans were obtained at a resolution of $2 \mathrm{~cm}^{-1}$, from 4000 to $400 \mathrm{~cm}^{-1}$ on a Shimadzu IR Prestige-21 equipment.

\subsubsection{Differential Scanning Calorimetry (DSC) analyses}

The samples, previously lyophilized (lipid to sugar molar ratio, 1:20) were placed in aluminum pans and heated from $15^{\circ} \mathrm{C}$ to $250^{\circ} \mathrm{C}$ at a rate of $10^{\circ} \mathrm{C} / \mathrm{min}$, under nitrogen pressure of $3 \mathrm{kgf} / \mathrm{cm}^{2}$ using a Jade Perkin Elmer equipment. Calibration was performed using indium and $\mathrm{n}$-octadecane as reference materials. In the experiment, it was ensured that the method had enough sensitivity to detect the amount of loaded drugs.

\subsubsection{Immunoliposomes development}

The functionalization of liposomes containing docetaxel followed the method previously described by our group, with modifications (Petrilli et al. 2017; Eloy et al. 2017). Initially, liposomes of SPC: CHOL: DSPE-PEG-Maleimide compositions were prepared for drug encapsulation, using the same protocol previously described. In parallel, the cetuximab antibody 
was diluted in PBS buffer $\mathrm{pH} 8.0$ containing EDTA $(5 \mathrm{mM})$ and the $\mathrm{pH}$ of the solution was adjusted to 8.0 with $\mathrm{NaOH}(0.1 \mathrm{~N})$. To the cetuximab solution, $5 \mathrm{mg} / \mathrm{mL}$, Traut's reagent solution ( $2 \mathrm{mg} / \mathrm{mL}$ ) was added, in a 40: 1 molar ratio (Traut's reagent:antibody). This solution was incubated for $1 \mathrm{~h}$ at $37{ }^{\circ} \mathrm{C}$ to allow thiolation. The separation of the thiolated antibody from the excessive Traut's reagent was carried out on gel filtration chromatography, using a PD-10 column, with eluent PBS-EDTA pH 8.0. After determining the concentration of cetuximab in each aliquot by BCA assay (bicinchoninic acid), the fractions containing the antibody were pooled and added to the liposomes previously prepared. The mixture was incubated overnight and finally the immunoliposome was purified on a CL-4B column to separate the liposome and free antibody, eluting using PBS buffer, $\mathrm{pH}$ 7.4. The immunoliposomes were further characterized by size, PdI and DTX encapsulation efficiency.

\subsubsection{Antibody conjugation efficiency}

The immunoliposome was separated from the free antibody by gel filtration chromatography with Sepharose CL-4B as stationary phase and PBS buffer (pH 7.4) as a mobile phase. The efficiency of cetuximab conjugation to liposomes was determined by the BCA assay, following the manufacturer's protocol (ThermoScientific). The conjugation efficiency was calculated by the percentage of antibody quantified in the immunoliposome in relation to the total antibody used in the experiment (Petrilli et al. 2017; Eloy et al. 2017).

\subsubsection{Antibody integrity after conjugation}

The integrity of the antibody in the immunoliposomes was assessed both by electrophoresis in polyacrylamide gel (SDS-PAGE) for its primary structure and for its tertiary structure using a fluorescence assay with intrinsic probes. Briefly, the SDS-PAGE electrophoresis was conducted according to the procedure previously described following the manufacturer's protocol (Eloy et al. 2017). The samples were previously incubated with 2-mercapto-ethanol and Laemmli sample buffer at $60{ }^{\circ} \mathrm{C}$ for $10 \mathrm{~min}$ for reduction. Mini-PROTEAN electrophoretic chamber and running buffer containing Tris / glycine / SDS were used for electrophoresis at 175 V for 30 min. The protein standard used was Precision Plus Dual Color Protein. After staining with Coomassie blue dye, the gels were discolored with deionized water and photographed.

For local tertiary structure investigation, antibody samples both in buffer (cetuximab) or immunoliposomes were analyzed by its intrinsic fluorescence emission spectra aiming to investigate possible interactions and modifications in protein tertiary conformation after cetuximab linkage to the liposomes. The analyses were carried out using a Hitachi F-4500 
spectrophotometer with excitation at $280 \mathrm{~nm}$ and emission between 300 and $420 \mathrm{~nm}$. Guanidine hydrochloride $\left(6 \mathrm{mmol} . \mathrm{L}^{-1}\right)$ was used as a denaturing agent in order to evaluate the suppression of fluorescence and the displacement of the maximum wavelength $\left(\lambda_{\max }\right)$ to less energetic wavelengths (Eftink 1994).

\subsubsection{In vitro drug release}

Liposomes and immunoliposomes containing the drug were evaluated for the release in $50 \mathrm{~mL}$ of phosphate buffer (PBS) containing $1.25 \%$ sodium lauryl sulfate as previously checked for the sink conditions, with stirring at $150 \mathrm{rpm}$ and $37^{\circ} \mathrm{C}$. For this study, samples were diluted in $1 \mathrm{~mL}$ of the buffer, at $\mathrm{pH} 7.4$, and placed in dialysis bags of $12-14 \mathrm{kDa}$ (limit molecular weight) (Yoon et al. 2017). Samples were collected in the receptor medium at different times, up to $48 \mathrm{~h}$. Subsequently they were filtered and analyzed by the HPLC method.

\subsubsection{Cell culture studies}

DU145 cells (high EGFR-expressing cells) were cultured in DMEM medium supplemented with $10 \% \mathrm{FBS}$ and $1 \%$ antibiotic / antimycotic solution, at $37^{\circ} \mathrm{C}$ with $5 \% \mathrm{CO}_{2}$, according to ATCC recommendations. PC-3 cells (low EGFR-expressing cells) (Kharmate et al., 2016) were cultured using the RPMI medium, supplemented with $10 \%$ FBS and $1 \%$ antibiotic / antimycotic solution. Once $90 \%$ of confluence was reached, the cells were trypsinized.

\subsubsection{Cellular uptake}

Liposomes and immunoliposomes uptake were assessed by confocal microscopy and flow cytometry studies. For this purpose, the formulations were prepared without drugs, containing the fluorescent lipid bilayer marker, DiO, encapsulated at $0.5 \mathrm{~mol} \%$. The preparation proceeded in the same way as previously specified for liposomes and immunoliposomes. The experiments were carried out with the cell line PC-3 (low EGFR-expressing cell line) and DU145 (EGFR positive cell line). Thus, for confocal studies, $1 \times 10^{6}$ cells/well were plated in 6-well microplates, containing sterile $22 \mathrm{~mm} / 22 \mathrm{~mm}$ coverslips and incubated for $24 \mathrm{~h}$ at $37^{\circ} \mathrm{C}$ in an atmosphere containing 5\% $\mathrm{CO}_{2}$. Then, the cells were washed with PBS buffer $\mathrm{pH} 7.4$ and incubated with the samples $(100 \mu \mathrm{L})$ diluted in incomplete medium for $2 \mathrm{~h}$ or $4 \mathrm{~h}$. After treatment, the cells were washed with PBS buffer and then $2 \mathrm{~mL}$ of $1 \%$ paraformaldehyde was added to the wells. The plates were kept overnight at $4{ }^{\circ} \mathrm{C}$, sealed with film. Then, the wells were again washed with PBS buffer and the coverslips were poured into histology slides containing Prolong 
Diamond ${ }^{\circledR}$ with DAPI, to preserve fluorescence and core labeling. The slides were visualized in a Leica TCS SP 8 confocal microscope $\left(\lambda_{\text {exc }}=405 \mathrm{~nm}, \lambda_{\mathrm{em}}=413-472 \mathrm{~nm}\right.$ (DAPI) and $\lambda_{\mathrm{exc}}=488$ $\mathrm{nm}, \lambda_{\mathrm{em}}=503-588 \mathrm{~nm}(\mathrm{DiO})$, increase of 63 times with oil immersion objective. For flow cytometry, the formulations were labelled with Dil. Liposomes were incubated in presence of 1 mol\% of Dil at $37^{\circ} \mathrm{C}$ for 1 hour and then purified using a PD-10 column. Cell uptake was monitored by flow cytometry using the fluorescent signal of Dil $(\lambda \mathrm{ex}=549 \mathrm{~nm}, \lambda \mathrm{em}=565 \mathrm{~nm})$ after incubation with the formulations. Cells were seeded in 12 -well plates $\left(1 \times 10^{5}\right.$ cells/well, 1 $\mathrm{mL} /$ well). Next day, liposomes or immunoliposomes, were diluted in $400 \mu \mathrm{L}$ of media and added to the cells. Cell uptake was monitored up to 3 hours after exposure to the formulations. The supernatant was aspirated and discarded, and the cells were washed twice with PBS. Cells were detached from the plates using a solution of $0.05 \%$ Trypsin/EDTA. Cells were washed with PBS and immediately acquired on the cytometer (BD FACSCalibur ${ }^{\mathrm{TM}}$, Becton, Dickinson and Company, UK). At least 10000 cells were counted for each sample, and experiments were performed in triplicates. Flow cytometry data were generated using BD CellQuest Pro software. The results are expressed as average \pm SD $(n=3)$. Statistical analysis was performed using Twoway ANOVA followed by Bonferroni post-test.

\subsubsection{Cytotoxicity}

PC-3 and DU145 cells were seeded at a seeding density of $1 \times 10^{4}$ cells/well in polysterene 96-well plates (Nunclon, Thermo Fisher Scientific, UK) in complete RPMI 1640 media. Next day, DTX, Lip-DTX or Lip-DTX-Ab were diluted in serum-free and antibiotics-free media and added to the cells. Untreated cells were used as a $100 \%$ viability control. After $6 \mathrm{~h}$ incubation, media containing liposomes were removed, cells were washed with PBS and replenished with fresh media supplements with 10\% (v/v) FBS and 1\% (v/v) antibiotics. At $24 \mathrm{~h}$ and $48 \mathrm{~h}$ post-incubation, a resazurin cell viability assay was performed, which is based on the mitochondrial metabolic activity of live cells. Resazurin reagent was prepared as previously described (Walzl et al., 2014). Briefly, cells were incubated with $0.01 \mathrm{mg} / \mathrm{mL}$ resazurin solution for $4 \mathrm{~h}$. After incubation, fluorescence $(\lambda \mathrm{ex}=544 \mathrm{~nm}$, $\lambda \mathrm{em}=590 \mathrm{~nm})$ was read using an automated FLUOstar Omega (BMG Labtech, UK) plate reader. Six replicates per condition were used. The results were expressed as the percentage of cell viability (mean $\pm \mathrm{SD}$ ) and normalized to control untreated cells (Pereira et al., 2016). The results were analyzed by two-way ANOVA, followed by Bonferroni post-test. 


\section{RESULTS AND DISCUSSION}

Liposomes are lipid vesicles widely used for the encapsulation of drugs, with several advantages, including protection against degradation, sustained release and improved pharmacokinetics. It is noteworthy, however, that several factors can affect the encapsulation of the drug, including the type of lipid, the ratio of drug to lipid, the presence of cholesterol and the PEGylation strategy for the long circulation time (Eloy et al., 2014b). An effective strategy for optimizing the composition of the release system is the use of a factorial planning, which rationalizes the development and allows the optimization of the number of experiments. This tool is part of the concept of "Quality by Design", which involves the definition of critical parameters that influence the attributes of the product, and is recommended by regulatory agencies in the drug development process (Singh, Sharma, and Robertson 2012; Troiano et al. 2016).

The experimental design matrix and the observed responses for the 15 experiments proposed by the Box-Behnken design produced ranges with maximum and minimum values for particle size ( $\left.\mathrm{Y}_{1}\right)$ (58.91 to $\left.108.63 \mathrm{~nm}\right)$, PdI $\left(\mathrm{Y}_{2}\right)$ (0.168 to 0.549$)$, and EE\% ( $\left.\mathrm{Y}_{3}\right)$ of, (68.56 to 99.45) as shown in Table 1.

Table 1: Box Behnken Design and Experimental responses.

\section{$<$ Table 1>}

From Figure 1 it was concluded that the variables studied did not adversely affect the particle size, considering that it remained below $100 \mathrm{~nm}$, with no statistical significance for the different observed particle size values. Soema and collaborators, on the other hand, concluded that the type of lipid (1,2-dioleoyl-sn-glycero-3-phosphoethanolamine (DOPE), 1,2-dioleoyl-3trimethylammoniumpropane (DOTAP) and 33-[N-(N0,N0-dimethylaminoethane)carbamoyl]cholesterol (DC-Chol)), and also the quantity can affect the particle size in liposomes prepared by extrusion (Soema et al. 2015). In addition, cholesterol, important for membrane fluidity and better physicochemical stability, has been reported to cause an increase in the liposome diameter (Shaker, Gardouh, and Ghorab 2017). Furthermore, it is known that the nanometric particle size influences the biological characteristics of the nanocarrier. For example, nanoparticles are captured and accumulated inside solid tumors by the EPR effect, due to the increased permeability of tumor blood vessels and deficient lymphatic clearance (Maeda et al. 2000).

The polydispersity index (PdI) reveals the homogeneity of particle size distribution, and the closer to 1.0 the PdI value, the more heterogeneous is the population of particles. Previous works have already reported PdI values close to 0.3 , thus in agreement with the values observed 
for the different formulations studied (Paini et al. 2015; Eloy et al. 2016). The results presented in Figure 1 revealed that particularly high proportions of cholesterol and DSPE-PEG resulted in an increase in PdI, since these lipids participate interfere in the formation of the bilayer.

Finally, the last stage of the development of liposomes containing DTX employing factorial design corresponded to the analysis of the influence of the variables on drug encapsulation. The results presented in Figure 1 showed that the efficiency of encapsulation of DTX docetaxel was high, ranging from $68.53 \%$ to $99.45 \%$, being the main contribution given by the lipid to drug ratio. As noted, encapsulation efficiency increased at lower molar concentrations of DTX. For the drug paclitaxel, for example, it has already been shown that high proportions of drug can result in its crystallization, due to the saturation of the lipid bilayer, impairing (Koudelka and Turánek 2012). Previously, it has been shown that liposomes composed of fluid, unsaturated lipid (DOPC:Chol:DSPE-PEG2000) showed the highest DTX encapsulation compared to rigid, saturated lipids (DPPC:Chol:DSPE-PEG2000 and DSPC:Chol:DSPE-PEG2000). Additionally, the inclusion of PEG showed a minimum effect on DTX encapsulation (Pereira et al., 2016).

Figure 1. Surface response graphs of DTX-loaded liposomes employing lipid/drug molar ratio, cholesterol/lipid ratio and DSPE-PEG (2000) molar ratio as independent variables and particle size $(\mathrm{nm})$, polydispersity index (PdI) and encapsulation efficiency (EE\%) as dependent variables.

<Figure 1>

A final quadratic polynomial equation was obtained to each response used to predict values and optimize parameters as shown in Eq. (3), (4) and (5). The polynomial equations were useful to interpret the results. A positive term means a synergistic effect (favors the optimization), whereas a negative term represents an antagonistic effect. In quadratic regression equations of response $Y_{1}$, the terms $X_{1}, X_{3}, X_{2}^{2} X_{3}^{2}$ had a negative influence which denoted a decrease in main effects of particle size. The terms $\mathrm{X}_{1}, \mathrm{X}_{1} \mathrm{X}_{2} \mathrm{X}_{2}^{2} \mathrm{e} \mathrm{X}_{3}^{2}$ for PdI presented the same behavior (negative influence). For $Y_{3}$, the positive relationship is observed in $X_{1}, X_{2}, X_{3}$ and $X_{3}^{2}$ terms which denoted an increase in the response. The $X_{1}, X_{2}$ and $X_{3}$ described represents results obtained for changing one variable at a time. The terms $\mathrm{X}_{1} \mathrm{X}_{2}, \mathrm{X}_{1} \mathrm{X}_{3}$, and $\mathrm{X}_{2} \mathrm{X}_{3}$ showed the changes when the variables simultaneously interact (Patil, Khairnar, and Naik 2015; Das and Mishra 2017). The quadratic model of responses $Y_{1}, Y_{2}$ and $Y_{3}$ generated the correlation coefficient that indicates an adequate fit $\left(\mathrm{R}^{2}\right)$. The coefficient values were found to be $0.6394,0.8269$ and 0.8491 , respectively. These results are in agreement with the study of Vergara et al., 2019 where liposomes were loaded with lactoferrin and their experimental design was used to optimize the responses of encapsulation efficiency and particle size for oral application. The value of correlation coefficient was 0.971 for 
$\mathrm{EE}$ and 0.841 for particle size represented adequate model for prediction from experimental data (Vergara and Shene 2019).

$$
\begin{aligned}
& Y_{1}=90.7267-2.2225 X_{1}+4,7587 X_{2}-1.9687 X_{3}+0.9575 X_{1} X_{2}+1.4475 X_{1} X_{3}+ \\
& 9.2350 X_{2} X_{3}+0.4192 X_{1}^{2}-15.7183 X_{2}^{2}-6.3933 X_{3}^{3} \\
& Y_{2}=0.437-0.009375 X_{1}+0.03525 X_{2}+0.019125 X_{3}-0.0005 X_{1} X_{2}+ \\
& 0.07125 X_{1} X_{3}+0.062 X_{2} X_{3}+0.000125 X_{1}^{2}-0.110125 X_{2}^{2}-0.092375 X_{3}^{3} \\
& Y_{3}=91.9533+8.5103 X_{1}+1.4035 X_{2}+2.4236 X_{3}-0.6850 X_{1} X_{2}-2.3954 X_{1} X_{3}- \\
& 1.0033 X_{2} X_{3}-10.3987 X_{1}^{2}-2.0862 X_{2}^{2}+0.8712 X_{3}^{3}
\end{aligned}
$$

The results presented in Figure 02 showed the normal probability distribution and fitted values considering residual values that had been calculated based on a predicted response and an experimental result. When the residual value did not present normal distribution, the residuals follow an approximate straight line which is used to calculate the cumulative distribution function for a specific population. As observed from the figure, the errors are normally distributed except for the probability plot in PdI response, whose confidence intervals can be affected. Overall, the model obtained had a satisfactory normal distribution, for particle size and encapsulation efficiency, particularly. The pattern between residuals and predicted responses in the plots of fitted values showed residues randomly dispersed (Wu et al. 2012; Hassan et al. 2019).

Figure 2 - Normal probability plot of residuals and residual in fitted value for (a) particle size Y1 (b) polydispersity index - Y2 and (c) percentage drug encapsulation - Y3.

\section{$<$ Figure 2>}

The ideal formulation that would have the lowest values of particle size and polydispersity index, combined with the best encapsulation efficiency, was then mathematically predicted. Such formulation corresponded to the composition with lipid: drug ratio, 23: 1, cholesterol: lipid ratio, 1.1: 10, and DSPE-PEG: lipid ratio, 0.75: 10. This formulation was further characterized, with particle size values of $67.47 \mathrm{~nm} \pm 4.32$, PdI of $0.287 \pm 0.006$, zeta potential of $-16.6 \mathrm{mV} \pm 0.6$ and encapsulation efficiency equivalent to $99.95 \%$, therefore the formulation revealed to be suitable for further studies. Furthermore, results presented as supplementary material show the measured and predicted values of the response considering the desirability function. The result for optimization was near to 1 indicating the suitability of the equation for the experimental data. 
Overall, the measured values obtained were satisfactory with the values calculated from the Box Behnken design. The percentage of relative error was calculated for each response. The EE\% $\left(\mathrm{Y}_{3}\right)$ showed the most adequate adjustment for the model obtained (Sudhakar, Krishna, and Murthy 2016; Vergara and Shene 2019).

Freeze-dried liposomes were characterized with respect to Fourier transform infrared spectroscopy (FTIR), compared to the blank liposome and the pure drug. FTIR analysis is important for the identification of the chemical groups present in the drug and carrier, and can be useful to show intermolecular interactions, such as hydrogen bonds, through changes in spectral peaks (Eloy et al. 2016). The main peak attributed to docetaxel corresponded to the vibration of the $\mathrm{C}=\mathrm{O}$ ester bond, present in approximately $1725 \mathrm{~cm}^{-1}$ (Keum et al. 2011). As shown in Figure $3 \mathrm{C}$, the spectra of the blank liposome and the liposome containing docetaxel were identical, so the peaks attributed to the drug could not be observed, which may be related to the dispersion of the drug encapsulated with molecular solution, rather than crystalline dispersion.

Calorimetric analyzes are also useful to elucidate the state of dispersion of the drug in the carrier, whether molecular, crystalline or amorphous, in addition to being useful to evidence intermolecular interactions. As seen in Figure 3D, the drug showed an endothermic peak at 166.37 ${ }^{\circ} \mathrm{C}$, with delta $\mathrm{H}$ equivalent to $27.7372 \mathrm{~J} / \mathrm{g}$, attributed to a crystalline substance. It was also noted that the DSC profile of the blank liposome was identical to that of the liposome with the encapsulated drug. The disappearance of the drug's endothermic peak in the liposome could be attributed to the inhibition of crystallization during the formation of the liposome, resulting in molecular or amorphous dispersion, similarly to that observed in polymeric nanoparticles containing docetaxel (Musumeci et al. 2006). In this way, the FTIR and DSC results, together, evidenced the reduction of DTX crystallinity.

Figure 3. Stability study of colloidal dispersion of DTX-loaded liposome and freeze-dried liposomes: evaluation of particle size (A) and polydispersity index (B). Solid state characterization of freeze-dried liposomes: FTIR (C) and DSC (D).

\section{$<$ Figure 3>}

The functionalization efficiency values of cetuximab evaluated by the quantification of antibody in the liposome fractions, separated by gel filtration chromatography, in relation to the total antibody, corresponded to $53.33 \%$ and $53.29 \%$, respectively for the immunoliposomes prepared with the concentration lowest and highest molar DSPE-PEG-MAL. Therefore, the concentration of maleimide investigated did not influence the functionalization of the liposome with cetuximab. Recently, Petrilli and collaborators synthesized immunoliposomes composed of 
dysteroylphosphatidylcholine (DSPC) and CHOL and functionalized by the tioeter bond with cetuximab, observing 59.4\% efficiency in the functionalization of the blank immunoliposome, similar to the result obtained in the present study (Petrilli et al. 2017).

The physicochemical characterization of the immunoliposome showed particle size and PdI corresponding to $128.8 \pm 2.35 \mathrm{~nm}$ and $0.279 \pm 0.002$, respectively, while the zeta potential was $-7.51 \pm 0.50$. It is noteworthy that these values were similar to those found in previous work of immunoliposome of similar composition, based on SPC: Chol: DSPE-MAL, containing the antibody trastuzumab (Eloy et al. 2017). Finally, the DTX encapsulation efficiency corresponded to $93.5 \pm 2.3 \%$ in the immunoliposome, showing that the functionalization reaction did not compromise the drug encapsulation.

As seen in Figure 4A, cetuximab exhibited two bands on the electrophoresis gel under reducing conditions: approximately $55 \mathrm{kDa}$ and $25 \mathrm{kDa}$, corresponding to the immunoglobulin heavy and light chains respectively. The electrophoretic profile of the immunoliposome was identical to that of cetuximab, so it is concluded that there were no damages in the protein and impairment of its primary structural integrity, as previously observed in liposomes functionalized with cetuximab (Petrilli et al. 2017)

In order to investigate the integrity of the tertiary structure of the antibody after functionalization, fluorescence assay was performed using intrinsic probes, that are, the aromatic amino acid residues present in the cetuximab structure. The tests were carried out at $280 \mathrm{~nm}$, in which the tryptophans and tyrosines were excited, and, thus, presenting considerable fluorescence intensity in that wavelength (Eftink, M. R., 1994). Figure 4B depicts the fluorescence emission spectra at four tested conditions: cetuximab thiol, cetuximab control and the two previous situations in the presence of $6 \mathrm{mmol} \cdot \mathrm{L}^{-1}$ of $\mathrm{GdnHCl}$ (Guanidine hydrochloride), a denaturant agent. Cetuximab presented the maximum emission wavelength $\left(\lambda_{\max }\right)$ centered at $336 \pm 1 \mathrm{~nm}$ indicating that the tryptophans, the main fluorophore, are mostly protected from the solvent. The chemically denaturation led cetuximab to suffer a red shift $\left(\lambda_{\max }\right.$ at $\left.350 \mathrm{~nm}\right)$ and fluorescence suppression indicating the tryptophans are now exposed to the solvent. Thiolated cetuximab, presented $\lambda_{\max }$ at around $340 \pm 1 \mathrm{~nm}$ indicating that the functionalization reaction caused a slightly red shift effect and a fluorescence suppression as well. These data could be an indicative that functionalization caused some change in the cetuximab local tertiary structure but did not cause denaturation. Thus, biological studies are paramount the investigate the maintenance of cetuximab biological role. 
Figure 4. Assessment of the integrity of (A) primary structure of antibody through SDS-PAGE electrophoresis (immunoliposome, IM, and cetuximab, CTX) and (B) integrity of tertiary structure through fluorescence spectroscopy of free antibody and functionalized, with or without incubation with denaturant molecule $\mathrm{GdnHCl}$.

\section{$<$ Figure 4>}

For the liposome and immunoliposome, DTX was released only after $12 \mathrm{~h}$ of study, with little difference in release between the times of $24 \mathrm{~h}$ and $48 \mathrm{~h}$, but being greater at $48 \mathrm{~h}$, as observed in figure 5B. It is evident that the liposome had a higher percentage of release compared to the immunoliposome, which can be explained due to the drug already strongly associated with the system, it still has the anchored antibody on its surface. For example, Zhang et al., 2005, developed liposomes containing paclitaxel, with a structure very similar to DTX, and obtained the peak of drug release, in PBS medium pH 7.4 similar to that used in this study, of $5.3 \%$ in approximately $48-50 \mathrm{~h}$, due to the drug being associated in a very stable way within the system. Haeri et al. 2016, developed thermosensitive liposomes (functionalized or not with cetuximab) containing doxorubicin and observed that at $37^{\circ} \mathrm{C}$, the same temperature used in this experiment, the maximum release was approximately $15 \%$ for both formulations, results similar to those observed in this work.

Figure 5. (A) TEM image of non-targeted liposome (a, scale bar of $500 \mathrm{~nm}$ ), immunoliposome (b, scale bar of 100nm) and immunoliposomes showing a field with more vesicles (c, scale bar of 200nm) and (B) in vitro release study of DTX-loaded liposome and immunoliposome using dialysis membrane.

\section{$<$ Figure 5>}

Drug encapsulation and targeting not only serve to increase drug cytotoxicity, but also targeted nanocarriers with monoclonal antibodies are able to increase the uptake by cells that overexpress the receptor to the antibody, as an alternative to decrease drug delivery to healthy tissues, thus avoiding side-effects (Eloy et al. 2017). In the present work, the cellular uptake of liposomes and immunoliposomes was evaluated in both prostate cancer cells PC-3 (with low EGFR expression) and DU145 (with high EGFR expression) (Sherwood et al. 1998). As expected, the results showed higher uptake of the immunoliposomes in the DU145 cells compared to the non-targeted liposome. Figure 6 showed that the uptake was low in both cell lines, PC-3 and 
DU145, both for the liposome and for the immunoliposome, after 2 or 4 hours of incubation. However, after $4 \mathrm{~h}$, the fluorescence of the encapsulated dye bordering the cell nucleus of the DU145 cells treated with the immunoliposome was very evident, but not for the low-EGFR expressing PC-3 cell line. Thus, we observed that increasing the incubation time from 2 to $4 \mathrm{~h}$ enhanced the uptake of immunoliposomes, but not liposomes, in the cell line that has higher levels of EGFR expression, DU145. We further confirmed the better uptake of immunoliposomes by the EGFR-positive cell line using flow cytometry technique. Immunoliposomes uptake was higher than the liposomes and was noticeable even after 30 minutes, increasing over time, resulting in almost a statistically significant 2-fold higher uptake of immunoliposomes than liposomes after 180 minutes in the DU 145 cell line, as shown in Figure 6D. These results are in agreement with previous reports where the role of the nanocarrier incubation time in cell uptake has been demonstrated. For example, liposomes functionalized with trastuzumab had greater cell internalization when incubated with breast cancer cells for 2 or 4 hours, than for 1 hour (Yang, Choi, et al. 2007).

The results of better delivery of immunoliposomes to EGFR-expressing cells are in agreement with the literature, for other cancer line cells. For example, in EGFR-overexpressing gastric cancer, cetuximab functionalization of polymeric nanoparticles improved DTX delivery, with a consequent increase in uptake and cytotoxicity, while, in vivo, the formulation resulted in efficient arrest of tumor growth compared to non-targeted nanoparticle, an aspect that will be investigated in future studies to be carried out by our group (Sreeranganathan et al. 2017). In the present study, we report for the first time, that cetuximab functionalization of DTX-loaded liposomes resulted in a higher uptake of the nanocarrier to EGFR-positive prostate cancer cells which could have a significant impact on improving drug delivery.

Figure 6. Cell uptake of liposomes and immunoliposomes by confocal microscopy and flow cytometry. In figure A, upper images I and II represent liposomes and immunoliposomes incubated in for $2 \mathrm{~h}$, while lower images III and IV correspond to $4 \mathrm{~h}$ incubation in low EGFRexpressing PC-3 cells. In figure B, upper images I and II represent liposomes and immunoliposomes incubated in for $2 \mathrm{~h}$, while lower images III and IV correspond to $4 \mathrm{~h}$ incubation in high EGFR-expressing DU145 cells. C represents a higher magnification of cells shown in figure B IV, highlighting cellular uptake of immunoliposomes. D: evaluation of liposome and immunoliposome uptake in EGFR-expressing DU145 cells at different incubation time-points by flow cytometry. The results are expressed as average \pm SD $(n=3)$. Statistical analysis was performed using Two-way ANOVA followed by Bonferroni post-test. * denotes comparison vs. the control $(* \mathrm{p}<0.05, * * \mathrm{p}<0.01, * * * \mathrm{p}<0.001, * * * * \mathrm{p}<0.0001)$ and \# denotes comparison between liposomes $(\# \mathrm{p}<0.05$, \#\# $\mathrm{p}<0.01$, \#\# $\mathrm{p}<0.001)$. 
$<$ Figure 6>

To investigate if the improved uptake of immunoliposomes would result in higher cytotoxicity, we incubated both PC-3 and DU145 cells for a short period of time and then washed the treatments and left the cells for 24 or $48 \mathrm{~h}$. The purpose of these experiments was to show in a clearer way the targeting effect of the antibody more than the non-specific uptake of the liposomes over a long period of time. The results, presented in the Figure 7 have shown that in PC-3 cell line, both DTX solution, DTX-loaded liposome and DTX-immunoliposome presented similar cytotoxicity. However, DTX-loaded immunoliposome was more cytotoxic than the free DTX and the DTX-loaded liposome in DU145 cell lines, due to the overexpression of EGFR, in agreement with the higher uptake shown herein. For instance, for the concentration of $100 \mathrm{nM}$ DTX, following $48 \mathrm{~h}$ of incubation, PC-3 and DU145 cell viability of the immunoliposome corresponded to, respectively, $68.71 \pm 2.34 \%$ and $40.58 \pm 6.99 \%$, whereas for the liposome the cell viability values in both cell lines were, respectively, $60.11 \pm 10.47 \%$ and $53.97 \pm 7.43 \%$. Detailed statistical comparisons are shown in supplementary material. It was observed statistical difference in only 4 comparisons in PC-3 treatment, mainly with lower DTX concentrations and reduced treatment time $(24 \mathrm{~h})$ for DTX vs immunoliposomes and liposomes vs immunoliposomes. Improved treatment efficacy for immunoliposomes was observed for DU145 cells, as it can be observed by a 3-fold increase in the number of statistically different comparisons, presented as supplementary material (12 comparisons for DU 145 vs 4 comparisons for PC-3 cells). Basically, DTX vs immunoliposomes presented statistical difference in all concentrations and treatment times tested in DU 145 (see supporting information, Table X?). Also, immunoliposomes showed better effect compared to liposomes at higher concentrations of DTX following $48 \mathrm{~h}$ treatment. The higher cytotoxicity of the immunoliposome against DU145 cells clearly indicated the role of cetuximab in promoting higher DTX cytotoxicity in a cell line with higher expression of EGFR. In a previous work, cetuximab showed a moderate response against DU145, whereas another line of prostate cancer, PC-3, was resistant (Dhupkar et al. 2010).

<Figure 7>

Figure 7. Cytotoxicity evaluation by resazurin assay of negative control, DTX, DTX-loaded liposomes and DTX-loaded immunoliposomes in PC3 and DU145 cells in different drug concentrations and incubation times. The results were analyzed by two-way ANOVA, with a 
Bonferroni test ( $p<0.05$ was considered the minimum value of significance) and the complete analysis was supplied as a supplementary material (Table X?).

\section{CONCLUSION}

Herein, docetaxel liposomes were developed using Box-Behnken factorial design. The developed liposomes were nano-sized with high loading efficiency and colloidal stability. Furthermore, the lyophilization process preserved liposomal physicochemical characteristics and solid-state characterizations were conducted showing that docetaxel was encapsulated with reduced crystallinity. Finally, the chosen formulation (with lipid: drug molar ratio, 23: 1, cholesterol: lipid molar ratio, 1.1:10, and DSPE-PEG:lipid molar ratio, 0.75:10) was conjugated with anti-EGFR antibody, cetuximab, with high efficiency, preserving the liposomal nanometric size and DTX high encapsulation. The integrity of anti-EGFR antibodies after the covalent linkage via maleimide chemistry to the liposomal surface was assessed and demonstrated primary and tertiary structure integrity. Both liposomes and immunoliposomes demonstrated DTX sustained release, in vitro. Also, biological in vitro cell culture studies were conducted in prostate cancer cell lines, with overexpression or not of the EGF receptor. Improved uptake was demonstrated due to receptor-ligand interactions in DU145 cell line compared with PC-3 cells. Besides, it was demonstrated that anti-EGFR liposomes were more effective than non-targeted nanoparticles in the EGFR-overexpressing cell line in terms of cytotoxicity. Thus, the developed immunoliposomes can be considered a potential platform for drug delivery of docetaxel to prostate cancer cells, with improved tumor cell selectivity and efficacy compared to non-targeted liposomes.

\section{ACKNOWLEDGEMENTS}

We thank FAPESP (Fundação do Amparo à Pesquisa do Estado de São Paulo) (projects \# 2016/02723-8, 2017/04091-1, 2018/00814-1 and 2018/21104-2), CNPQ (Conselho Nacional de Desenvolvimento Científico e Tecnológico (Project \# 409362/2018-2) and CAPES (Coordenação de Aperfeiçoamento de Pessoal de Nível Superior - Brasil) - Finance Code 001 for financial support. This work is part of the National Institute of Science and Technology in Pharmaceutical Nanotechnology: a transdisciplinary approach INCT-NANOFARMA. Dr AlJamal would like to thank Prostate Cancer UK (CDF12-002) for funding. 


\section{REFERENCES}

Cathomas, Richard, Christian Rothermundt, Dirk Klingbiel, Lukas Bubendorf, Rolf Jaggi, Daniel C. Betticher, Peter Brauchli, Denise Cotting, Cornelia Droege, Ralph Winterhalder, Daniele Siciliano, Dominik R. Berthold, Miklos Pless, Ralph Schiess, Roger von Moos, and Silke Gillessen. 2012. "Efficacy of Cetuximab in Metastatic Castration-Resistant Prostate Cancer Might Depend on EGFR and PTEN Expression: Results from a Phase II Trial (SAKK 08/07).” Cancer Therapy: Clinical 12: 6049-6057. DOI: 10.1158/1078-0432.CCR-12-2219.

Das, Shilpi, and Susmita Mishra. 2017. "Box-Behnken Statistical Design to Optimize Preparation of Activated Carbon from Limonia Acidissima Shell with Desirability Approach." Journal of Environmental Chemical Engineering 5 (1): 588-600. https://doi.org/10.1016/j.jece.2016.12.034.

Dhupkar, Pooja, Melissa Dowling, Keith Cengel, and Bin Chen. 2010. "Effects of AntiEGFR Antibody Cetuximab on Androgen-Independent Prostate Cancer Cells.” Anticancer Research 30 (6): 1905-10.

Eftink, M. R. 1994. "The Use of Fluorescence Methods to Monitor Unfolding Transitions in Proteins." Biochemistry (Moscow) 63 (3): 276-84.

Eloy, Josimar O., Raquel Petrilli, Deise L. Chesca, Fabiano P. Saggioro, Robert J. Lee, and Juliana Maldonado Marchetti. 2017. “Anti-HER2 Immunoliposomes for CoDelivery of Paclitaxel and Rapamycin for Breast Cancer Therapy." European Journal of Pharmaceutics and Biopharmaceutics 115 (4): 159-67. https://doi.org/10.1016/j.ejpb.2017.02.020.

Eloy, Josimar O., Raquel Petrilli, José Fernando Topan, Heriton Marcelo Ribeiro Antonio, Juliana Palma Abriata Barcellos, Deise L. Chesca, Luciano Neder Serafini, Daniel G. Tiezzi, Robert J. Lee, and Juliana Maldonado Marchetti. 2016. "CoLoaded Paclitaxel/Rapamycin Liposomes: Development, Characterization and in Vitro and in Vivo Evaluation for Breast Cancer Therapy." Colloids and Surfaces B: Biointerfaces 141: 74-82. https://doi.org/10.1016/j.colsurfb.2016.01.032.

Engels, Frederike K., Ron A.A. Mathot, and Jaap Verweij. 2007. "Alternative Drug Formulations of Docetaxel: A Review." Anti-Cancer Drugs 18 (2): 95-103. https://doi.org/10.1097/CAD.0b013e3280113338. 
Guardiola, Salvador, Monica Varese, and Macarena Sa. 2019. "Review A Third Shot at EGFR: New Opportunities in Cancer Therapy,” 1-15. https://doi.org/10.1016/j.tips.2019.10.004

Guérin, O, P. Formento, C, Lo Nigro, P. Hoffman, J.L. Fischel, M.C. Etienne-Grimaldi, M. Merlano, J.M. Ferrero, G. Milano. "Supra-additive antitumor effect of sunitinib malate (SU11248, Sutent ${ }^{\circledR}$ ) combined with docetaxel. A new therapeutic perspective in hormone refractory prostate cancer". Journal of Cancer Research and Clinical Oncology 134: 51-57, 10.1007/s00432-007-0247-4.

Haeri, Azadeh, Sara Zalba, Timo L.M. ten Hagen, Simin Dadashzadeh, and Gerben A. Koning. 2016. "EGFR Targeted Thermosensitive Liposomes: A Novel Multifunctional Platform for Simultaneous Tumor Targeted and Stimulus Responsive Drug Delivery." Colloids and Surfaces B: Biointerfaces 146: 657-69. https://doi.org/10.1016/j.colsurfb.2016.06.012.

Hassan, Mohamad Zaki, S. M. Sapuan, Siti Amni Roslan, Sa'Ardin Abdul Aziz, and Shamsul Sarip. 2019. "Optimization of Tensile Behavior of Banana Pseudo-Stem (Musa Acuminate) Fiber Reinforced Epoxy Composites Using Response Surface Methodology." Journal of Materials Research and Technology 8 (4): 3517-28. https://doi.org/10.1016/j.jmrt.2019.06.026.

Immordino, Maria Laura, Franco Dosio, and Luigi Cattel. 2006. "Stealth Liposomes: Review of the Basic Science, Rationale, and Clinical Applications, Existing and Potential.” International Journal of Nanomedicine 1 (3): 297-315.

Joshi, Gaurav, Pankaj Kumar Singh, Arvind Negi, Anil Rana, Sandeep Singh, and Raj Kumar. 2015. "Growth Factors Mediated Cell Signalling in Prostate Cancer Progression: Implications in Discovery of Anti-Prostate Cancer Agents." ChemicoBiological Interactions 240: 120-33. https://doi.org/10.1016/j.cbi.2015.08.009.

Kharmate, Geetanjali, Elham Hosseini-Beheshti, Josselin Caradec, Mei Yieng Chin, Emma S. Tomlinson Guns, 2015. "Epidermal Growth Factor Receptor in Prostate Cancer Derived Exosomes". PLOS ONE 11(5):e0154967. doi:10.1371/ journal.pone.0154967.

Keum, Chang Gu, Young Wook Noh, Jong Suep Baek, Ji Ho Lim, Chan Ju Hwang, Young Guk Na, Sang Chul Shin, and Cheong Weon Cho. 2011. "Practical 
Preparation Procedures for Docetaxel-Loaded Nanoparticles Using Polylactic AcidCo-Glycolic Acid." International Journal of Nanomedicine 6: 2225-34. https://doi.org/10.2147/ijn.s24547.

Koudelka, Štěpán, and Jaroslav Turánek. 2012. "Liposomal Paclitaxel Formulations." Journal of Controlled Release $163 \quad$ (3): 322-34. https://doi.org/10.1016/j.jconrel.2012.09.006.

Li, Mingyuan, Chunyang Du, Na Guo, Yuou Teng, Xin Meng, Hua Sun, Shuangshuang Li, Peng Yu, and Hervé Galons. 2019. "Composition Design and Medical Application of Liposomes." European Journal of Medicinal Chemistry 164: 64053. https://doi.org/10.1016/j.ejmech.2019.01.007.

Maeda, H., J. WU, T. Sawa, Y Matsumura, and K Hori. 2000. "Tumor Vascular Permeability and the EPR Effect in Macromolecular Therapeutics: A Review." Journal of Controlled Release 65: 271-284.

Mager, Rene, Olga Savko, Katharina Böhm, Anita Thomas, Robert Dotzauer, Hendrik Borgmann, Wolfgang Jäger, et al. 2019. “Comparative Assessment of Docetaxel for Safety and Efficacy between Hormone-Sensitive and Castration-Resistant Metastatic Prostate Cancer." Urologic Oncology: Seminars and Original Investigations 37 (12): 999-1005. https://doi.org/10.1016/j.urolonc.2019.07.005.

Malmberg, Jennie, Vladimir Tolmachev, and Anna Orlova. 2011. "Imaging Agents for in Vivo Molecular Profiling of Disseminated Prostate Cancer - Targeting EGFR Receptors in Prostate Cancer: Comparison of Cellular Processing of [111In]Labeled Affibody Molecule ZEGFR:2377 and Cetuximab." International Journal of Oncology 38 (4): 1137-43. https://doi.org/10.3892/ijo.2011.915.

Martins, Susana, Bruno Sarmento, Domingos C. Ferreira, and Eliana B. Souto. 2007. “Lipid-Based Colloidal Carriers for Peptide and Protein Delivery - Liposomes versus Lipid Nanoparticles.” International Journal of Nanomedicine 2 (4): 595-607.

Merino, María, Sara Zalba, and María J. Garrido. 2018. "Immunoliposomes in Clinical Oncology: State of the Art and Future Perspectives." Journal of Controlled Release 275 (2017): 162-76. https://doi.org/10.1016/j.jconrel.2018.02.015.

Moraes, Carolina M., Eneida De Paula, André H. Rosa, and Leonardo F. Fraceto. 2010. "Physicochemical Stability of Poly(Lactide-Co-Glycolide) Nanocapsules 
Containing the Local Anesthetic Bupivacaine." Journal of the Brazilian Chemical Society 21 (6): 995-1000. https://doi.org/10.1590/S0103-50532010000600008.

Musumeci, T., C. A. Ventura, I. Giannone, B. Ruozi, L. Montenegro, R. Pignatello, and G. Puglisi. 2006. "PLA/PLGA Nanoparticles for Sustained Release of Docetaxel." International Journal of Pharmaceutics $325 \quad$ (1-2): 172-79. https://doi.org/10.1016/j.ijpharm.2006.06.023.

Nabila, Morshed, Nishat Jahan, and Diandra Elizabeth Penheiro. 2018. "Polymeric Nanoparticles for Targeted Delivery in Cancer Treatment: An Overview." International Journal of Pharmaceutical Sciences Review and Research 52 (1): 101$11 . \quad \mathrm{http}: / /$ globalresearchonline.net/journalcontents/v521/19.pdf\%0Ahttp://ovidsp.ovid.com/ovidweb.cgi?T=JS\&PAGE=reference \&D=em exa\&NEWS=N\&AN=624202638.

Nelson, William G, Emmanuel S Antonarakis, H Ballentine Carter, Angelo M De Marzo, and Theodore L Deweese. n.d. Prostate Cancer. Abeloff's Clinical Oncology. Sixth Edit. Elsevier Inc. https://doi.org/10.1016/B978-0-323-47674-4.00081-5.

Paini, Marco, Sean Ryan Daly, Bahar Aliakbarian, Ali Fathi, Elmira Arab Tehrany, Patrizia Perego, Fariba Dehghani, and Peter Valtchev. 2015. “An Efficient Liposome Based Method for Antioxidants Encapsulation." Colloids and Surfaces B: Biointerfaces 136: 1067-72. https://doi.org/10.1016/j.colsurfb.2015.10.038.

Patel, Jitendrakumar, Jitendra Amrutiya, Priyanka Bhatt, Ankit Javia, Mukul Jain \& Ambikanandan Misra. 2018. Journal of microencapsulation 35, 204-217. https://doi.org/10.1080/02652048.2018.1453560.

Patil, Pritam, Gokul Khairnar, and Jitendra Naik. 2015. "Preparation and Statistical Optimization of Losartan Potassium Loaded Nanoparticles Using Box Behnken Factorial Design: Microreactor Precipitation.” Chemical Engineering Research and Design 104: 98-109. https://doi.org/10.1016/j.cherd.2015.07.021.

Pereira, Sara, Rapahel Egbu, Gemma Jannati, and Wafa' T. Al-Jamal. 2016. "Docetaxelloaded liposomes: The effect of lipid composition and purification on drug encapsulation and in vitro toxicity." International Journal of Pharmaceutics 514:150-159. https://doi.org/10.1016/j.ijpharm.2016.06.057.

Petrilli, Raquel, Josimar Eloy, Renata Lopez, and Robert Lee. 2017. "Cetuximab 
Immunoliposomes Enhance Delivery of 5-FU to Skin Squamous Carcinoma Cells.” Anti-Cancer Agents in Medicinal Chemistry 17: 301-8. https://doi.org/10.2174/1871520616666160526110913.

Petrilli, Raquel, Josimar O. Eloy, Fabiano P. Saggioro, Deise L. Chesca, Marina Claro de Souza,Marcos V.S. Dias, LuisL.P. da Silva, Robert J. Lee, Renata F.V.Lopez. 2018.

" Skin cancer treatment effectiveness is improved by iontophoresis of EGFRtargeted liposomes containing 5-FU compared with subcutaneous injection." Journal of Controlled Release 283:151-162. https://doi.org/10.1016/j.jconrel.2018.05.038

Rawla, Prashanth. 2019. "Epidemiology of Prostate Cancer." World Journal of Oncology 10 (2): 63-89. https://doi.org/10.1159/000423644.

Rodallec, Anne, Jean Michel Brunel, Sarah Giacometti, Helene Maccario, Florian Correard, Eric Mas, Caroline Orneto, et al. 2018. "Docetaxel-Trastuzumab Stealth Immunoliposome: Development and in Vitro Proof of Concept Studies in Breast Cancer." International Journal of Nanomedicine 13: 3451-65. https://doi.org/10.2147/IJN.S162454.

Saad, Fred, and Kurt Miller. 2014. "Treatment Options in Castration-Resistant Prostate Cancer: Current Therapies and Emerging Docetaxel-Based Regimens." Urologic Oncology: Seminars and Original Investigations 32 (2): 70-79. https://doi.org/10.1016/j.urolonc.2013.01.005.

Shaker, Sherif, Ahmed Gardouh, and Mamdouh Ghorab. 2017. "Factors Affecting Liposomes Particle Size Prepared by Ethanol Injection Method." Research in Pharmaceutical Sciences 12 (5): 346-52. https://doi.org/10.4103/17355362.213979.

Sherwood, E. R., J. L. Van Dongen, C. G. Wood, S. Liao, J. M. Kozlowski, and C. Lee. 1998. "Epidermal Growth Factor Receptor Activation in Androgen-Independent but Not Androgen-Stimulated Growth of Human Prostatic Carcinoma Cells." British Journal of Cancer 77 (6): 855-61. https://doi.org/10.1038/bjc.1998.142.

Singh, Sanjay, Arati Sharma, and Gavin P. Robertson. 2012. "Realizing the Clinical Potential of Cancer Nanotechnology by Minimizing Toxicological and Targeted Delivery Concerns.” Cancer Res. 72 (22): 5663-68. https://doi.org/10.1158/00085472.CAN-12-1527. 
Soema, Peter C., Geert Jan Willems, Wim Jiskoot, Jean Pierre Amorij, and Gideon F.

Kersten. 2015. "Predicting the Influence of Liposomal Lipid Composition on Liposome Size, Zeta Potential and Liposome-Induced Dendritic Cell Maturation Using a Design of Experiments Approach.” European Journal of Pharmaceutics and Biopharmaceutics 94: 427-35. https://doi.org/10.1016/j.ejpb.2015.06.026.

Solanki, Ajay B., Jolly R. Parikh, and Rajesh H. Parikh. 2007. "Formulation and Optimization of Piroxicam Proniosomes by 3-Factor, 3-Level Box-Behnken Design.” AAPS PharmSciTech 8 (4). https://doi.org/10.1208/pt0804086.

Sreeranganathan, Maya, Saji Uthaman, Bruno Sarmento, Chethampadi Gopi Mohan, In Kyu Park, and Rangasamy Jayakumar. 2017. "In Vivo Evaluation of CetuximabConjugated Poly $(\gamma$-Glutamic Acid)-Docetaxel Nanomedicines in EGFROverexpressing Gastric Cancer Xenografts.” International Journal of Nanomedicine 12: 7167-82. https://doi.org/10.2147/IJN.S143529.

Sudhakar, Beeravelli, Mylangam Chaitanya Krishna, and Kolapalli Venkata Ramana Murthy. 2016. "Factorial Design Studies of Antiretroviral Drug-Loaded Stealth Liposomal Injectable: PEGylation, Lyophilization and Pharmacokinetic Studies." Applied Nanoscience (Switzerland) 6 (1): 43-60. https://doi.org/10.1007/s13204015-0408-8.

Troiano, Greg, Jim Nolan, Donald Parsons, Christina Van Geen Hoven, and Stephen Zale. 2016. “A Quality by Design Approach to Developing and Manufacturing Polymeric Nanoparticle Drug Products.” AAPS Journal 18 (6): 1354-65. https://doi.org/10.1208/s12248-016-9969-z.

Tucci, Marcello, Orazio Caffo, Consuelo Buttigliero, Carla Cavaliere, Carmine D'aniello, Massimo Di Maio, Stefania Kinspergher, et al. 2019. “Therapeutic Options for FirstLine Metastatic Castration-Resistant Prostate Cancer: Suggestions for Clinical Practise in the CHAARTED and LATITUDE Era." Cancer Treatment Reviews 74 (January): 35-42. https://doi.org/10.1016/j.ctrv.2019.01.002.

Vergara, Daniela, and Carolina Shene. 2019. "Encapsulation of Lactoferrin into Rapeseed Phospholipids Based Liposomes: Optimization and Physicochemical Characterization." Journal of Food Engineering 262 (February): 29-38. https://doi.org/10.1016/j.jfoodeng.2019.05.012. 
Wu, Long, Kit Lun Yick, Sun Pui Ng, and Joanne Yip. 2012. "Application of the BoxBehnken Design to the Optimization of Process Parameters in Foam Cup Molding." Expert Systems with Applications $39 \quad$ (9): 8059-65. https://doi.org/10.1016/j.eswa.2012.01.137.

Yang, Tao, Min Koo Choi, Fu De Cui, Jung Sun Kim, Suk Jae Chung, Chang Koo Shim, and Dae Duk Kim. 2007. "Preparation and Evaluation of Paclitaxel-Loaded PEGylated Immunoliposome." Journal of Controlled Release 120 (3): 169-77. https://doi.org/10.1016/j.jconrel.2007.05.011.

Yang, Tao, Fu De Cui, Min Koo Choi, Jei Won Cho, Suk Jae Chung, Chang Koo Shim, and Dae Duk Kim. 2007. "Enhanced Solubility and Stability of PEGylated Liposomal Paclitaxel: In Vitro and in Vivo Evaluation." International Journal of Pharmaceutics 338 (1-2): 317-26. https://doi.org/10.1016/j.ijpharm.2007.02.011.

Yoon, Ho Yub, Seong Shin Kwak, Moon Ho Jang, Min Hyung Kang, Si Woo Sung, Chang Hyun Kim, Sung Rae Kim, Dong Woo Yeom, Myung Joo Kang, and Young Wook Choi. 2017. "Docetaxel-Loaded RIPL Peptide (IPLVVPLRRRRRRRRC)Conjugated Liposomes: Drug Release, Cytotoxicity, and Antitumor Efficacy." International Journal of Pharmaceutics 523 (1): 229-37. https://doi.org/10.1016/j.ijpharm.2017.03.045.

Zalba, Sara, Ana M. Contreras, Azadeh Haeri, Timo L.M. Ten Hagen, Iñigo Navarro, Gerben Koning, and María J. Garrido. 2015. “Cetuximab-Oxaliplatin-Liposomes for Epidermal Growth Factor Receptor Targeted Chemotherapy of Colorectal Cancer.” Journal of Controlled Release 210: 26-38. https://doi.org/10.1016/j.jconrel.2015.05.271. 